\title{
TAXONOMY OF THE GENUS Paris L. (Melanthiaceae) IN VIETNAM
}

\author{
Nguyen Quynh Nga*, Pham Thanh Huyen, Phan Van Truong, Hoang Van Toan \\ National Institute of Medicinal Materials
}

\begin{abstract}
Paris L. is a small genus distributed widely in Eurasia. In Vietnam Paris occur in evergreen broad-leaved forests in some mountainous areas of the North and the Central highlands. Due to over-exploitation as well as habitat loss, populations of some Paris species are seriously declining. This genus has not been studied extensively in Vietnam. The aim of this study was to define the morphological characteristics of the genus Paris in Vietnam. Morphological description, dichotomous key for identification, ecology and distributions of the genus in Vietnam are reported. The results show that this genus in Vietnam comprises 8 species and 2 varieties, possesing unilocular ovary with parietal placenta.
\end{abstract}

Keywords: Melanthiaceae, Paris, identification key, morphological description, Vietnam.

Citation: Nguyen Quynh Nga, Pham Thanh Huyen, Phan Van Truong, Hoang Van Toan, 2016. Taxonomy of the genus Paris L. (Melanthiaceae) in Vietnam. Tap chi Sinh hoc, 38(3): 333-339. DOI: 10.15625/0866$7160 / \mathrm{v} 38 \mathrm{n} 3.8400$.

*Corresponding author: nguyenquynhnga@hotmail.com.

\section{INTRODUCTION}

The genus Paris L. (Melanthiaceae), which is locally known as Bay la mot hoa or Trong lau, is a small genus distributed in evergreen broadleaved forests of some mountainous areas in the North and the Central highlands of Vietnam. Originally this genus comprised 5 species in Vietnam [3]. Then, Do (2007) [1] recorded 6 species after a first extensive study. However, ovaries of those species in her work were erroneously described as multilocular with axile placentation instead of unilocular with parietal placentation, as mentioned in the orginial description as well as other relevant works $[2$, 6]. Recently, 2 more new species i.e. $P$. cronquistii (Takht.) $\mathrm{H}$. Li and P. xichouensis $(\mathrm{H}$. Li) Y. H. Ji, H. Li \& Z. K. Zhou. $[4,5]$ have been recorded and the total number of Paris species in Vietnam is now 8 species with 2 varieties. The aim of this study is to define the morphological characteristics and to construct taxonomic key of the genus Paris in Vietnam.

\section{MATERIALS AND METHODS}

Fresh samples of the genus Paris were collected from the field in the Northern and Central Vietnam, 76 voucher specimens were deposited in the herbarium of the National Institute of Medicinal Materials (NIMM).
Morphological characters of all collected samples were analyzed and taxonomic key was prepared with reference to the relevant documents $[1,2,6]$.

\section{RESULTS AND DISCUSSION}

Paris L. - Bảy lá một hoa (Trọng lâu)

L. 1753. Sp. Pl. 367; J. Hutch. 1959. Fam. Fl. Pl. ed. 2.2: 615; F. T. Wang \& al. in F. T. Wang \& T. Tang, 1978. Fl. Reip. Pop. Sin. 15: 86-96; Takht. 1983. Brittonia, 35: 255-270; H. Li 1984. Act. Bot. Yunn. 6(4): 351-362; id. 1986. Bull. Bot. Res. 6910: 109-144; id. 1986. Guihaia, 6(3): 187-192: N. T. Ban \& N. T. Do, 1992. Journ. Biol. 14 (4): 5; N. T. Do, 1992. Journ. Pharm. 6: 10; id. 1995. Journ. Biol. 17(4, special vol); 128; Takht. 1996. Div. Class. Fl. Pl. 485; Tamura in Kubitzki, 1998. Fam. Gen. Vasc. Pl. 3: 450; S. Liang \& V. G. Soukup in Z. Y. Wu \& P H. Raven, 2000. Fl. China, 24: 88-95; N. T. Do, 2007. Fl. Viet. 6: 10.

Type: Paris quadrifolia $\mathrm{L}$.

Herbs perennial, terrestrial part 0.5-2 m, erect, solitary, unbranched. Rhizome horizontal, cylindrical, grow underground, send out roots and shoots from their nodes. Leaves 4 to many, in a terminal whorl, petiolate; lanceolate, ovate to obovate; green, abaxially with or without purple 
blotches; papery or leathery, glabrous; main veins 3-7 with anastomosing veinlets; apex acute to acuminate; base cuneate obtuse, truncate to cordate. Flowers bisexual, solitary, developing from the top of stem, pedunculate. Peduncle 10$80 \mathrm{~cm}$. Tepals in 2 whorls, free. Outer tepals 36(8) nearly as many as leave and inner tepal number; the number of outer tepals may vary within a species; green to yellow-green; ovate to lanceolate; apex acute to acuminate. Inner tepals 3-6 (7-8), the number of inner tepals may vary among species, nearly as many as leave and outer tepal number; longer, equal or slightly shorter or much shorter than the outer ones; green, yellowgreen or dark purple; linear or spatulate, apex acute; reflexed-bent backward or not. Stamens in
1-2 whorls, nearly 2-3 times as many as the number of leaves, outer and inner tepals. The number of stamens may vary among species. Filaments shorter or equal to anthers. Free portion of connective transversely ellipsoid to subglose or cylindric with apex rounded or acuminate. Ovary subglobose or ellipsoid, shallowly or deeply ribbed, horizontal cross section square to polygon with convex or concave sides; transverse rim blue violet, violet, purple to red orange; unilocular with parietal placenta; ovules numerous, arranged along placentas. Style nearly equal to or shorter than stigma, stigma lobes 3-7(8). Fruit capsule, loculicidal, many seeded. Seed subglobose or ellipsoid, aril fleshy.
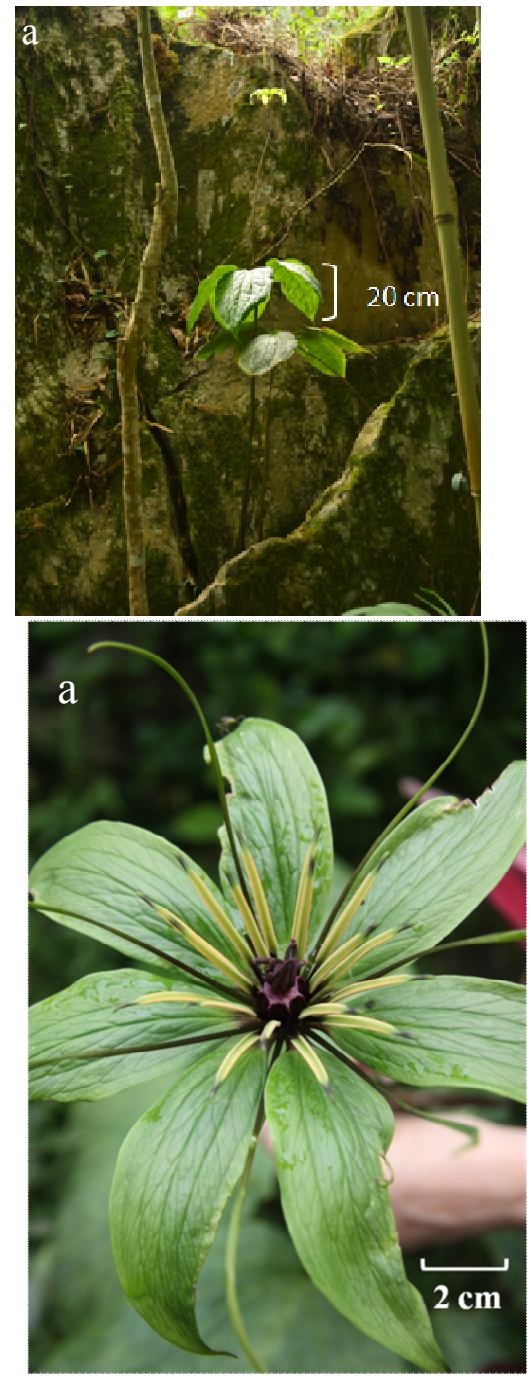

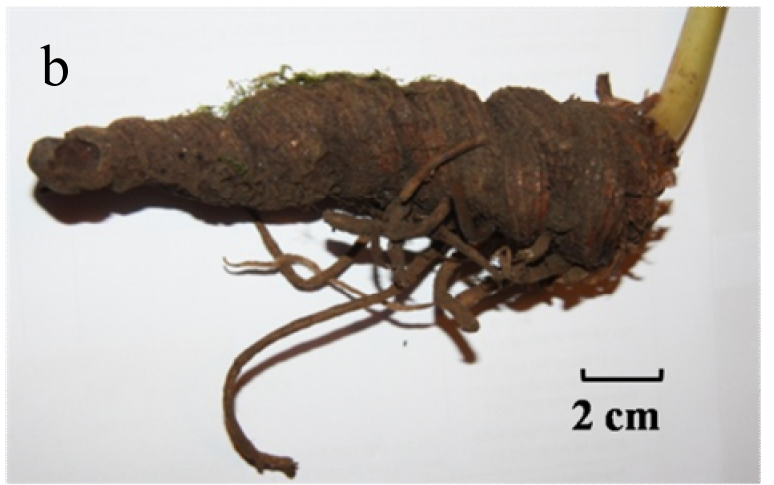

Figure 1. (a) Habit and (b) rhizome of P. xichouensis (H. Li) Y. H. Ji, H. Li \& Z. K. Zhou

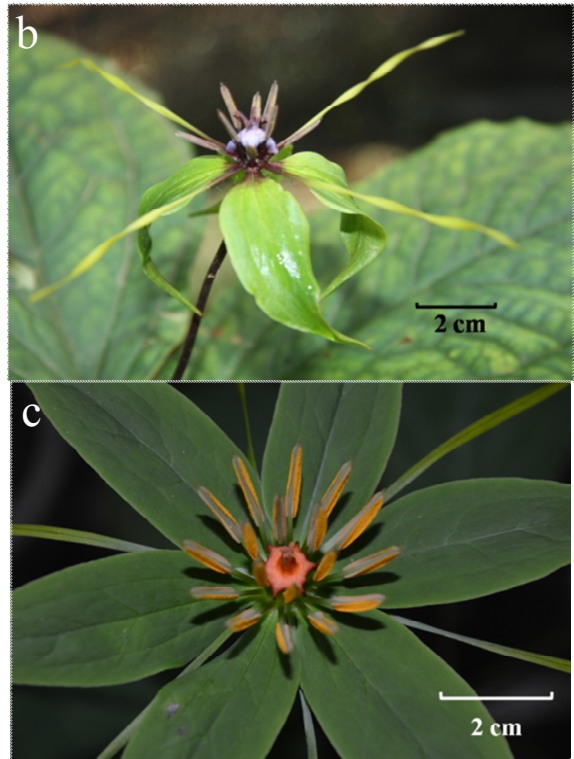



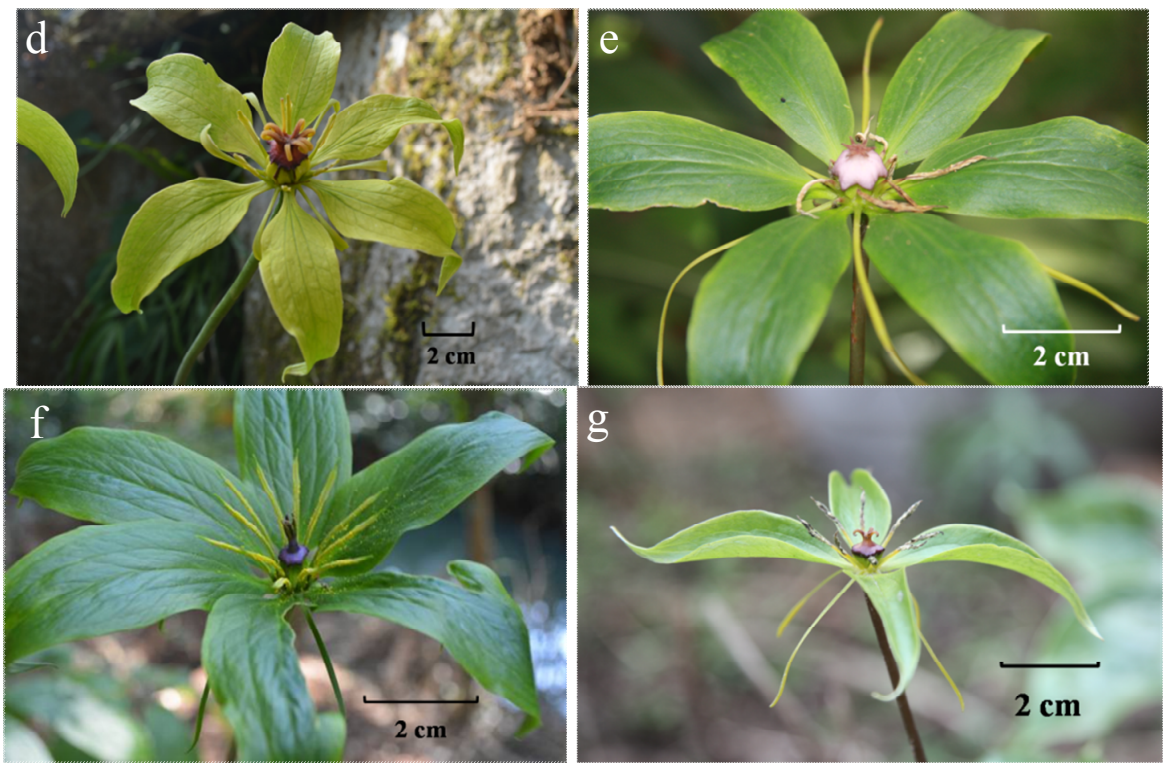

Figure 2. Flowers of some Paris species

a. P. dunniana H. Lév. ; b. P. vietnamensis (Takht) H. Li; c. P. cronquistii (Takht.) H. Li; d. P. xichouensis (H. Li) Y. H. Ji, H. Li \& Z. K. Zhou; e. P. polyphylla var. yunnanensis; f. P. polyphylla var. chinensis (Franch.) H. Hara; g. P. caobangensis Y. H. Ji, H. Li \& Z. K. Zhou
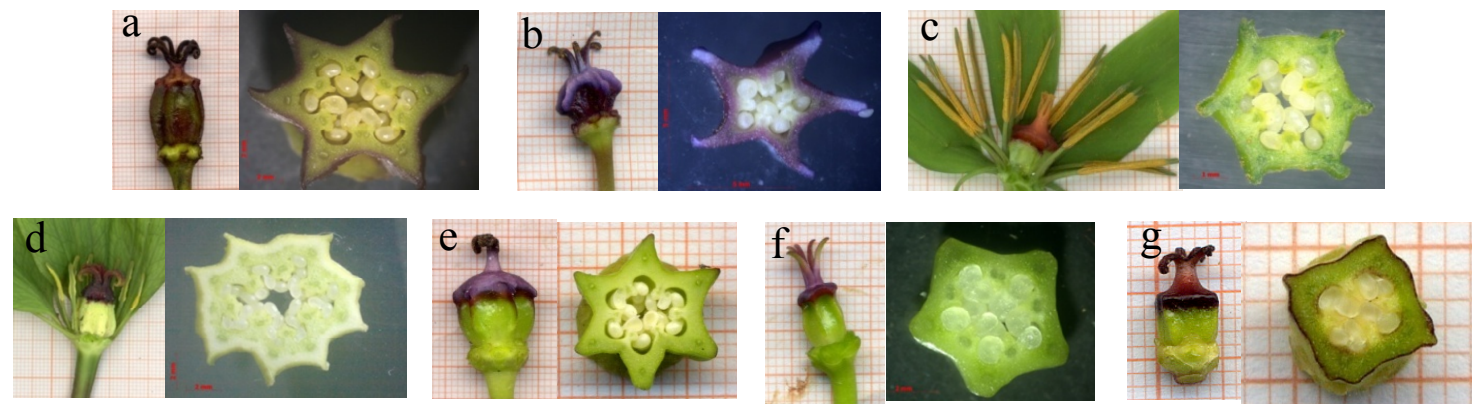

Figure 3. Gynoecium (left) and ovary horizontal cross section (right) of some Paris species a. P. dunniana H.Lév. ; b. P. vietnamensis (Takht) H.Li; c. P. cronquistii (Takht.) H.Li; d. P. xichouensis (H.Li) Y.H.Ji, H.Li \& Z.K.Zhou; e. P. polyphylla var. yunnanensis; f. P. polyphylla var. chinensis (Franch.) H.Hara; g. P. caobangensis Y.H.Ji, H.Li \& Z.K.Zhou
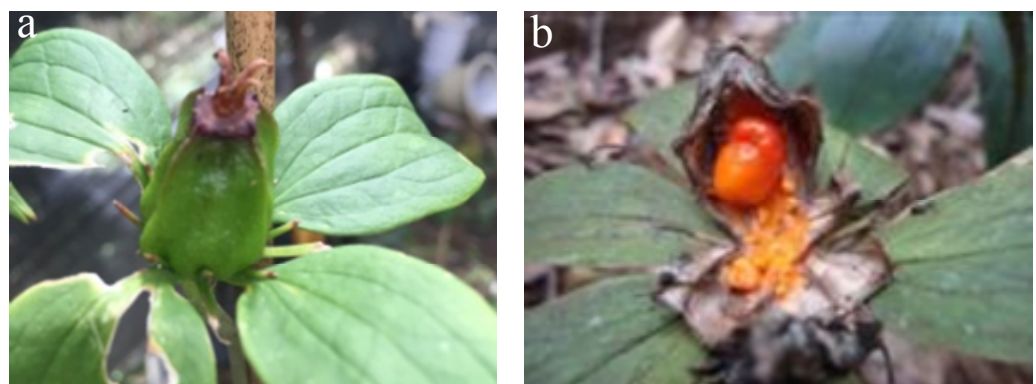

Figure 4. a. Young capsule of P. xichouensis (H.Li) Y.H.Ji, H.Li \& Z.K.Zhou; b. Dehiscent capsule of P. polyphylla var. chinensis (Franch.) H.Hara 
Distribution, ecology and medicinal uses of Paris species in Vietnam

Ecology: Paris species mostly grow on moist soil, near stream, limestone slopes or under the canopy in evergreen forests; elevation 600-1,500 m. Fl. April-June, fr. July-November.

Distribution: Distribution of Paris species in Vietnam is shown in Fig. 5.

P. caobangensis Y. H. Ji, H. Li \& Z. K. Zhou: Lao Cai prov. (Sa Pa), Cao Bang (Nguyen Binh); P. cronquistii (Takht.) H. Li: Ha Giang prov. (Dong Van); P. delavayi Franch.: Lao Cai prov. (Sa Pa), Ha Giang prov. (Dong Van), Cao Bang prov. (Nguyen Binh), Vinh Phuc prov. (Tam Dao).; P. dunniana $\mathrm{H}$. Lév.: Lao Cai prov. (Sa Pa), Vinh Phuc prov. (Tam Dao), Ninh Binh prov. (Cuc Phuong), Kon Tum (Kon Plong).; P. fargesii Franch.: Ha Noi (Ba Vi), Thanh Hoa prov. (Quan Hoa, Ba Thuoc); P. polyphylla var. yunnanensis (Franch.) Hand.-Mazz.: Lai Chau prov. (Phong Tho), Ha Noi (Ba Vi), Thanh Hoa prov. (Ba Thuoc); $P$. polyphylla var. chinensis (Franch.) H. Hara: Lao Cai prov. (Sa Pa), Yen Bai (Nghia Lo), Dien
Bien prov. (Tuan Giao), Phu Tho prov. (Thanh Son), Ha Noi (Ba Vi), Hoa Binh prov. (Luong Son, Mai Chau), Ninh Binh prov. (Cuc Phuong), Nghe An prov. (Con Cuong), Quang Nam prov. (Nam Giang), Quang Binh prov. (Bo Trach), Kon Tum prov. (Kon Plong); P. vietnamensis (Takht) H.Li: Ha Giang prov. (Hoang Su Phi, Xin Man), Lao Cai prov. (Sa Pa), Lai Chau prov. (Phong Tho), Vinh Phuc prov. (Tam Dao), Lam Dong prov. (Lac Duong); P. xichouensis (H. Li) Y. H. Ji, H. Li \& Z. K. Zhou: Ha Giang prov. (Dong Van) (picture 5).

Medicinal uses: In traditional medicine, rhizomes of Paris are valuable. They have been used to treat many diseases such as fever, malaria, snake bites, pimples, inflammation of the mammary gland, tuberculosis and asthma [8].

However, due to over-exploitation as well as habitat loss, populations of some Paris species are seriously declining. For example, $P$. cronquistii (Takht.) H. Li and P. xichouensis (H.Li) Y. H. Ji, H. Li \& Z. K. Zhou occur only in the narrow areas in Ha Giang province.

Table 1. Comparison of some morphological characters among Paris species in Vietnam

\begin{tabular}{|c|c|c|c|c|c|c|}
\hline $\mathrm{N}^{\mathrm{o}}$ & Morphological & Leave & Inner tepal & $\begin{array}{c}\text { Free portion } \\
\text { of anther } \\
\text { connective }\end{array}$ & Ovary & Style and stigma \\
\hline 1 & $\begin{array}{l}\text { P. caobangensis } \\
\text { Y. H. Ji, H. Li \& } \\
\text { Z. K. Zhou }\end{array}$ & $\begin{array}{l}\text { Abaxially } \\
\text { without } \\
\text { purple } \\
\text { blotches }\end{array}$ & $\begin{array}{l}\text { Yellow green, } \\
\text { linear, shorter } \\
\text { than outer one }\end{array}$ & $\begin{array}{l}\text { Cylindric, } \\
\text { apex } \\
\text { acuminate, } \\
2-2.5 \mathrm{~mm}\end{array}$ & $\begin{array}{l}4-5 \text { ribbed, } \\
\text { horizontal } \\
\text { cross } \\
\text { section (HS) } \\
\text { convex } \\
\text { polygon }\end{array}$ & $\begin{array}{l}\text { Style } \\
\text { conspicuous, } \\
\text { equal to stigma }\end{array}$ \\
\hline 2 & $\begin{array}{l}\text { P. cronquistii } \\
\text { (Takht.) H. Li }\end{array}$ & $\begin{array}{l}\text { Abaxially } \\
\text { with purple } \\
\text { blotches }\end{array}$ & $\begin{array}{l}\text { Yellow green, } \\
\text { linear, longer } \\
\text { than outer one }\end{array}$ & $\begin{array}{l}\text { Cylindrical, } \\
\text { apex } \\
\text { acuminate, } \\
1.5-2 \mathrm{~mm}\end{array}$ & $\begin{array}{l}5-6 \text { ribbed, } \\
\text { HS concave } \\
\text { polygon }\end{array}$ & $\begin{array}{l}\text { Style } \\
\text { conspicuous, } \\
\text { equal to stigma }\end{array}$ \\
\hline 3 & $\begin{array}{l}\text { P. delavayi } \\
\text { Franch. }\end{array}$ & $\begin{array}{l}\text { Abaxially } \\
\text { without } \\
\text { purple } \\
\text { blotches }\end{array}$ & $\begin{array}{l}\text { Purple, linear, } \\
\text { shorter than } \\
\text { outer one }\end{array}$ & $\begin{array}{l}\text { Cylindrical, } \\
\text { apex } \\
\text { acuminate, } \\
3-4 \mathrm{~mm}\end{array}$ & $\begin{array}{l}4-5 \text { ribbed } \\
\text { HS concave } \\
\text { polygon }\end{array}$ & $\begin{array}{l}\text { Style } \\
\text { conspicuous, } \\
\text { equal to stigma }\end{array}$ \\
\hline 4 & $\begin{array}{l}\text { P. dunniana } \mathrm{H} \text {. } \\
\text { Lév. }\end{array}$ & $\begin{array}{l}\text { Abaxially } \\
\text { without } \\
\text { purple } \\
\text { blotches }\end{array}$ & $\begin{array}{l}\text { Green, linear, } \\
\text { longer than } \\
\text { outer one }\end{array}$ & $\begin{array}{l}\text { Cylindrical, } \\
\text { apex } \\
\text { acuminate, } \\
4.0-4.5 \mathrm{~mm} \\
\end{array}$ & $\begin{array}{l}\text { 6-8 ribbed, } \\
\text { HS concave } \\
\text { polygon }\end{array}$ & $\begin{array}{l}\text { Style } \\
\text { conspicuous, } \\
\text { shorter than } \\
\text { stigma }\end{array}$ \\
\hline 5 & P. fargesii Franch. & $\begin{array}{l}\text { Abaxially } \\
\text { without } \\
\text { purple } \\
\text { blotches }\end{array}$ & $\begin{array}{l}\text { Yellow green, } \\
\text { linear, shorter } \\
\text { than outer one }\end{array}$ & $\begin{array}{l}\text { Transversely } \\
\text { ellipsoid, } \\
\text { subglobose; } \\
1.0-2.0 \mathrm{~mm}\end{array}$ & $\begin{array}{l}4-5 \text { ribbed } \\
\text { HS concave } \\
\text { polygon }\end{array}$ & $\begin{array}{l}\text { Style } \\
\text { cinconspicuous }\end{array}$ \\
\hline 6 & P. polyphylla Sm. & Abaxially & Yellow green to & Cylindrical, & 5-7 ribbed, & Style \\
\hline
\end{tabular}


Taxonomy of the genus Paris L. (Melanthiaceae)

\begin{tabular}{|c|c|c|c|c|c|c|}
\hline & & $\begin{array}{l}\text { without } \\
\text { purple } \\
\text { blotches }\end{array}$ & $\begin{array}{l}\text { green, linear, } \\
\text { shorter than (or } \\
\text { nearly equal to) } \\
\text { outer one, } \\
\text { reflexed or not } \\
\text { reflexed }\end{array}$ & $\begin{array}{l}\text { apex } \\
\text { acuminate, } \\
1.0-2.5 \mathrm{~mm}\end{array}$ & $\begin{array}{l}\text { HS concave } \\
\text { polygon }\end{array}$ & $\begin{array}{l}\text { conspicuous, } \\
\text { equal or shorter } \\
\text { than stigma }\end{array}$ \\
\hline & $\begin{array}{l}\text { P. polyphylla var. } \\
\text { yunnanensis } \\
\text { (Franch.) Hand.- } \\
\text { Mazz. }\end{array}$ & $\begin{array}{l}\text { Abaxially } \\
\text { without } \\
\text { purple } \\
\text { blotches }\end{array}$ & $\begin{array}{l}\text { Yellow green, } \\
\text { linear, shorter } \\
\text { than (or nearly } \\
\text { equal to) outer } \\
\text { one, not } \\
\text { reflexed }\end{array}$ & $\begin{array}{l}\text { Cylindrical, } \\
\text { apex } \\
\text { acuminate, } \\
1.0-1.5 \mathrm{~mm}\end{array}$ & $\begin{array}{l}\text { 5-7 ribbed, } \\
\text { HS concave } \\
\text { polygon }\end{array}$ & $\begin{array}{l}\text { Style } \\
\text { conspicuous, } \\
\text { equal to stigma }\end{array}$ \\
\hline & $\begin{array}{l}\text { P. polyphylla var. } \\
\text { chinensis } \\
\text { (Franch.) H. Hara }\end{array}$ & $\begin{array}{l}\text { Abaxially } \\
\text { without } \\
\text { purple } \\
\text { blotches }\end{array}$ & $\begin{array}{l}\text { Green, linear, } \\
\text { shorter than } \\
\text { outer one, } \\
\text { reflexed }\end{array}$ & $\begin{array}{l}\text { Cylindrical, } \\
\text { apex } \\
\text { acuminate, } \\
1.5-2.5 \mathrm{~mm}\end{array}$ & $\begin{array}{l}\text { 5-7 ribbed, } \\
\text { HS concave } \\
\text { polygon }\end{array}$ & $\begin{array}{l}\text { Style } \\
\text { conspicuous, } \\
\text { equal or shorter } \\
\text { than stigma }\end{array}$ \\
\hline 7 & $\begin{array}{l}\text { P. vietnamensis } \\
\text { (Takht) H.Li }\end{array}$ & $\begin{array}{l}\text { Abaxially } \\
\text { without } \\
\text { purple } \\
\text { blotches }\end{array}$ & $\begin{array}{l}\text { Yellow green, } \\
\text { linear, longer } \\
\text { than outer one }\end{array}$ & $\begin{array}{l}\text { Cylindrical, } \\
\text { apex } \\
\text { acuminate, } \\
1.0-1.5 \mathrm{~mm}\end{array}$ & $\begin{array}{l}\text { 4-6 deeply } \\
\text { ribbed, HS } \\
\text { star-shaped }\end{array}$ & $\begin{array}{l}\text { Style } \\
\text { inconspicuous }\end{array}$ \\
\hline 8 & $\begin{array}{l}\text { P. xichouensis }(\mathrm{H} . \\
\mathrm{Li}) \mathrm{Y} . \mathrm{H} . \mathrm{Ji}, \mathrm{H} . \mathrm{Li} \\
\text { \& Z. K. Zhou }\end{array}$ & $\begin{array}{l}\text { Abaxially } \\
\text { without } \\
\text { purple } \\
\text { blotches }\end{array}$ & $\begin{array}{l}\text { Yellow green } \\
\text { spatulate, } \\
\text { shorter than } \\
\text { outer one }\end{array}$ & $\begin{array}{l}\text { Cylindrical, } \\
\text { apex rounded, } \\
2.0-2.5 \mathrm{~mm}\end{array}$ & $\begin{array}{l}\text { 6-8 ribbed, } \\
\text { HS concave } \\
\text { polygon }\end{array}$ & $\begin{array}{l}\text { Style } \\
\text { inconspicuous }\end{array}$ \\
\hline
\end{tabular}

Identification key for the genus Paris L. in identification key of Li [2] and Liang \& Soukup Vietnam [6], a taxonomic key was constructed for the

Based on the personal observation and the genus Paris L. in Vietnam. It is presented hereunder:

1A. Free portion of anther connective transversely ellipsoid, subglobose

1. P. fagersii

1B. Free portion of anther connective cylindrical with apex rounded or acuminate

2A. Inner tepals $(4-11 \mathrm{~cm})$ longer than outer tepals $(2-7 \mathrm{~cm})$

3A. Ovary deeply ribbed, style inconspicuous.

2. P. vietnamensis

3B. Ovary ribbed, style conspicuous

4A. Leaves without purple blotches. Style $(2-3 \mathrm{~mm})$ shorter than stigma $(6-8 \mathrm{~mm}) \ldots .3$. P. dunniana 4B. Leave abaxially with purple blotches. Style and stigma nearly equal in

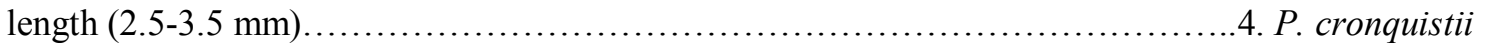

2B. Inner tepals $(2.5-7.5 \mathrm{~cm})$ shorter or nearly as long as outer tepals $(5-9 \mathrm{~cm})$

5 A. Free portion of anther connective apex rounded, inner tepals spatulate. 5. P. xichouensis

5B. Free portion of anther connective apex acuminate, inner tepals linear

6A. Inner tepal purple to dark purple, free portion of anther connective 3-4 mm 6. P. delavayii

6B. Inner tepal green, yellow green, free portion of anther connective 1-2.5 mm

7A. Ovary 4-5 ribbed, horizontal cross section convex polygon................... 7. P. caobangensis

7B. Ovary 5-7 ribbed, horizontal cross section of ovary concave polygon............8. P. polyphylla

$8 \mathrm{~A}$. Inner tepals slightly shorter or as long as outer tepals, not reflexed. 9. P. polyphylla var. yunnanensis

8B. Inner tepals much shorter than outer tepals, reflexed 10. P. polyphylla var. chinensis 


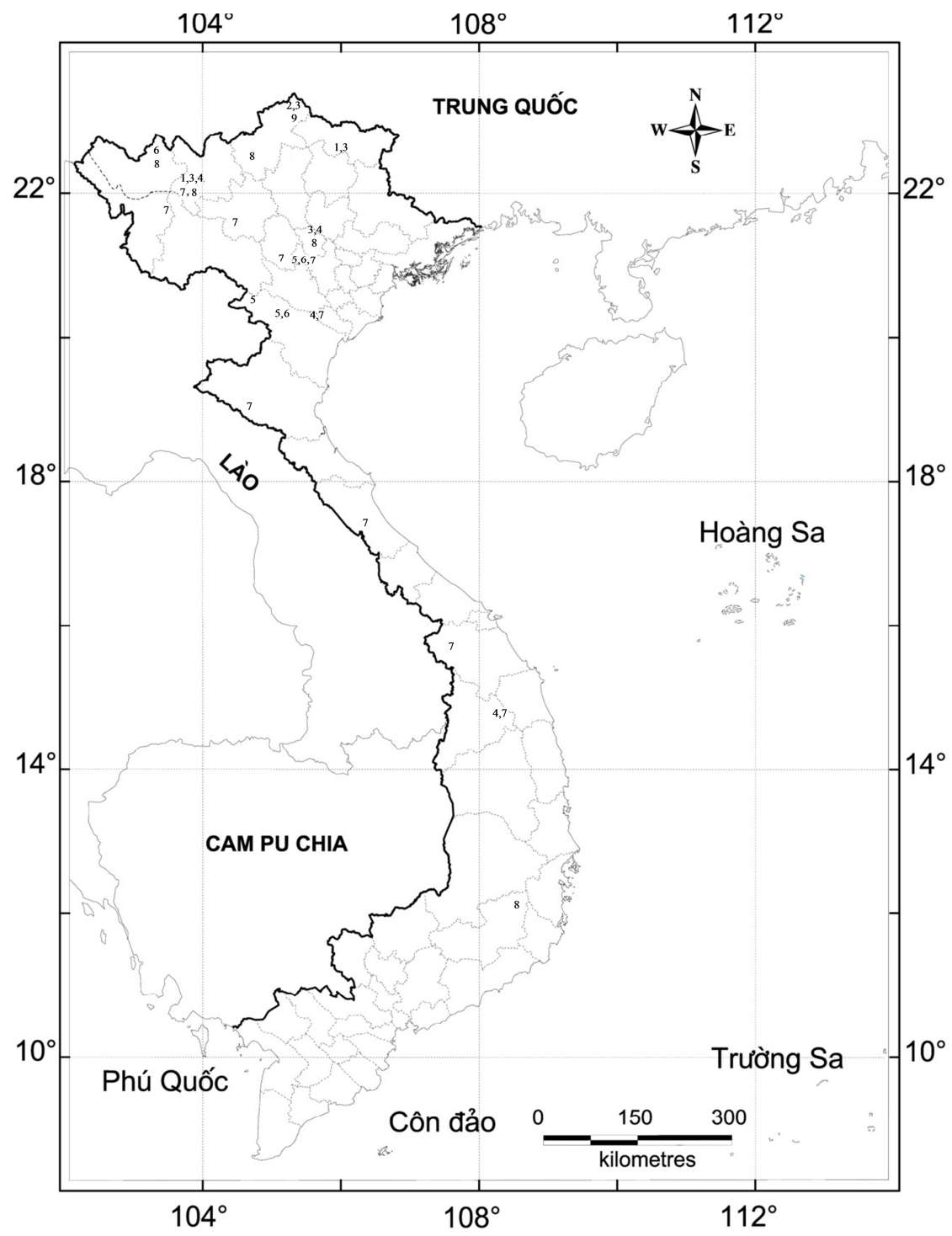

Picture 5. Distribution of Paris species in Vietnam

1. P. caobangensis Y. H. Ji, H. Li \& Z. K. Zhou; 2. P. cronquistii (Takht.) H. Li; 3. P. delavayi Franch.; 4. P. dunniana H. Lév.; 5. P. fargesii Franch.; 6. P. polyphylla var. yunnanensis (Franch.) Hand.-Mazz.; 7. P. polyphylla var. chinensis (Franch.) H. Hara; 8. P. vietnamensis (Takht) H.Li; 9. P. xichouensis (H. Li) Y. H. Ji, H. Li \& Z. K. Zhou

\section{CONCLUSIONS}

Morphological analysis of this study showed that all 8 species with 2 varieties of the genus Paris in Vietnam possess unilocular ovary with parietal placenta. Based on the characteristics of inner and outer tepal, free portion of connective apex, stamen, style and ovary, an identification key for the species of the genus Paris in Vietnam is constructed. Information of ecology, distributions and their uses are also reported additionally.

Acknowledgements: The results of this article is an outcome of the project: "Survey, collecting samples and identifying of some Paris species 
(Melanthiaceae) in Vietnam using morphological characteristics and PCR-RFLP markers" supported by National Institute of Medicinal Materials.

\section{REFERENCES}

1. Nguyen Thi Do, 2007. Trilliaceae. Thuc vat chi Viet Nam, Bo hoa Loa ken-Liliales Perleb. Science and Technics Publishing House, Ha Noi, 8: 311-321.

2. Heng Li, 1998. The phylogeny of the genus Paris L. In: Heng Li, editor. The genus Paris (Trilliaceae), Science Press, Beijing, pp. 8-65 (in Chinese).

3. Pham Hoang Ho, 2000. Cay co Viet Nam. Tre Publishing House, Ho Chi Minh city, 3: 474-475.

4. Nguyen Quynh Nga, Pham Thanh Huyen, Phan Van Truong, Hoang Van Toan, Nguyen Xuan Nam, 2015. A newly recorded species-Paris cronquistii (Takht.) $\mathrm{H}$. Li to flora of Vietnam. Journal of
Medicinal Materials, 4(20): 203-206 (in Vietnamese).

5. Nguyen Quynh Nga, Pham Thanh Huyen, Phan Van Truong, Hoang Van Toan, Nguyen Ngoc Cong, 2015. Paris xichouensis (H. Li) Y. H. Ji \& Z. K. Zhou-A newly recorded species-in the flora of Vietnam. Journal of Medicinal Materials, 5(20): 264-267.

6. Songyun Liang, Victor G. Soukup, 2000. Paris Linnaeus. In: Wu, Z. Y., P. H. Raven (eds.), Flora of China, Science Press, Beijing, and Missouri Botanical Garden Press, St. Louis, 24: 88-90.

7. Nguyen Nghia Thin, 2007. Cac phuong phap nghien cuu thuc vat. Nxb. Đại học Quốc gia, Hà Nội, tr. 23-27.

8. Vien Duoc Lieu, 2006. Cay thuoc va dong vat lam thuoc o Viet Nam, tap 1. Science and Technics Publishing House, Ha Noi: 182-184.

\section{NGHIÊN CỨU ĐĂC ĐIỂM HÌNH THÁI CỦA CHI Paris L. (Melanthiaceae) Ở VIẸT NAM}

\section{Nguyễn Quỳnh Nga, Phạm Thanh Huyền, Phan Văn Trưởng, Hoàng Văn Toán}

Khoa Tài nguyên dược liệu, Viện Dược liệu

\section{TÓM TẮT}

Paris L., thuộc họ Melanthiaceae, là một chi nhỏ, phân bố chủ yếu ở vùng núi cao từ các tỉnh phía Bắc cho đến Tây Nguyên. Nhiều loài thuộc chi Paris là những cây thuốc có giá trị, tuy nhiên, việc chặt phá rừng đã làm thu hẹp môi trường sống, cùng với tình trạng thu gom buôn bán bất hợp pháp trong những năm gần đây đã làm suy giảm nguồn dược liệu quí này. Cho đến nay, các công trình nghiên cứu về đặc điểm hình thái của chi Paris (Bảy lá một hoa) ở Việt Nam vẫn chưa đầy đủ. Trong nghiên cứu này, chúng tôi đã mô tả đặc điểm hình thái và xây dựng khóa định loại cho 8 loài và 2 thứ thuộc chi Paris ở Việt Nam; bài báo còn cung cấp một số thông tin về đặc điểm sinh thái, phân bố và giá trị sử dụng. Dựa vào các đăc điểm hình thái có thể kết luận các loài thuộc chi Paris ở Việt Nam đã thống kê được đều thuộc nhóm bầu 1 ô, noãn đính bên.

Từ khóa: Melanthiaceae, Paris, đặc điểm hình thái, khóa định loại, Việt Nam.

Received 13 June 2016, accepted 20 September 2016 